\title{
Photo-induced Metal-Catalyst-Free Aromatic Finkelstein Reaction
}

\author{
$\mathrm{Lu} \mathrm{Li}^{\dagger, \ddagger, \S}$ Wenbo Liu, ${ }^{\dagger, \S}$ Huiying Zeng, ${ }^{\dagger}$ Xiaoyue $\mathrm{Mu},{ }^{\dagger}$ Gonzalo Cosa, ${ }^{\dagger}$ Zetian Mi, ${ }^{*},{ }^{\ddagger}$ and Chao-Jun Li* ${ }^{\dagger}$ \\ ${ }^{\dagger}$ Department of Chemistry, McGill University, 801 Sherbrooke Street West, Montreal, Quebec H3A 0B8, Canada \\ ${ }^{\ddagger}$ Department of Electrical and Computer Engineering, McGill University, 3480 University Street, Montreal, Quebec H3A 0E9, \\ Canada
}

\section{Supporting Information}

ABSTRACT: The facile iodination of aromatic compounds under mild conditions is a great challenge for both organic and medicinal chemistry. Particularly, the synthesis of functionalized aryl iodides by light has long been considered impossible due to their photo-lability, which actually makes aryl iodides popular starting materials in many photo-substitution reactions. Herein, a photoinduced halogen exchange in aryl or vinyl halides has been discovered for the first time. A broad scope of aryl iodides can be prepared in high yields at room temperature under exceptionally mild conditions without any metal or photo-redox catalysts. The presence of a catalytic amount of elemental iodine could promote the reaction significantly.

A ryl and heteroaryl iodides are ubiquitous precursors and fundamental building blocks used in chemistry for many important transformations, such as metalation processes, ${ }^{1,2}$ nucleophilic aromatic substitutions, ${ }^{3}$ and transition-metalcatalyzed cross-coupling reactions. ${ }^{4,5}$ Furthermore, aryl iodides and their radiolabeled analogues have proven to be very useful in nuclear medicine and radiotherapy science, such as hypothyroidism treatment, ${ }^{6}$ single-photon-emission computed tomography, ${ }^{7}$ and preclinical X-ray imaging. ${ }^{8}$ The various radioactive iodine isotopes $\left({ }^{131} \mathrm{I},{ }^{125} \mathrm{I}\right.$, and $\left.{ }^{123} \mathrm{I}\right)$ make it possible to label the same target agent with different isotopes, increasing the flexibility in pharmacokinetic studies. ${ }^{9}$ However, compared to the less reactive aryl bromides and chlorides, the corresponding aryl iodides are much more difficult to obtain. ${ }^{10}$ The routine iodination methods usually result in poor yields or require stoichiometric amount of transition-metal reagents. ${ }^{11-13}$ Substrate scope also suffers from severe limitations due to the harsh reaction conditions. More recently, great efforts have been devoted and several useful strategies have been developed to synthesize functionalized (hetero)aromatic iodides with high yield and broad substrate scope. One of the major improvements has been made via the palladium-catalyzed $\mathrm{C}-\mathrm{H}$ bond iodination assisted by directing groups. ${ }^{14-18}$ Another pivotal progress is the copper- or nickel-catalyzed aryl halide exchange process assisted by ligands-the so-called aromatic Finkelstein reaction-which was first reported by Buchwald and co-workers. ${ }^{19-22}$

Despite the tremendous progresses achieved, the current transition-metal-catalyzed iodination methods are still not straightforward and atom economical enough, especially compared to the classical Finkelstein reaction, in which alkyl chlorides and bromides (aryl, vinyl, and tertiary alkyl halides are unreactive) can be converted into the corresponding alkyl iodides simply using a solution of sodium iodide in acetone. Furthermore, the difficulty associated with metal or ligand residue has led to growing concerns about the pharmaceuticals and materials. In this respect, a transition-metal-free iodination approach to access aryl iodides more efficiently and under milder and environmentally benign conditions is highly desired. ${ }^{23}$ As an emerging alternative to the metal-catalyzed strategy in catalysis, ${ }^{24-31}$ we envisioned that photo-energy might possess some unique reactivity toward the aromatic halogen exchange process. As shown in Scheme 1, we describe a novel aromatic Finkelstein reaction assisted by UV light at room temperature under very mild and metal-catalyst-free reaction conditions.

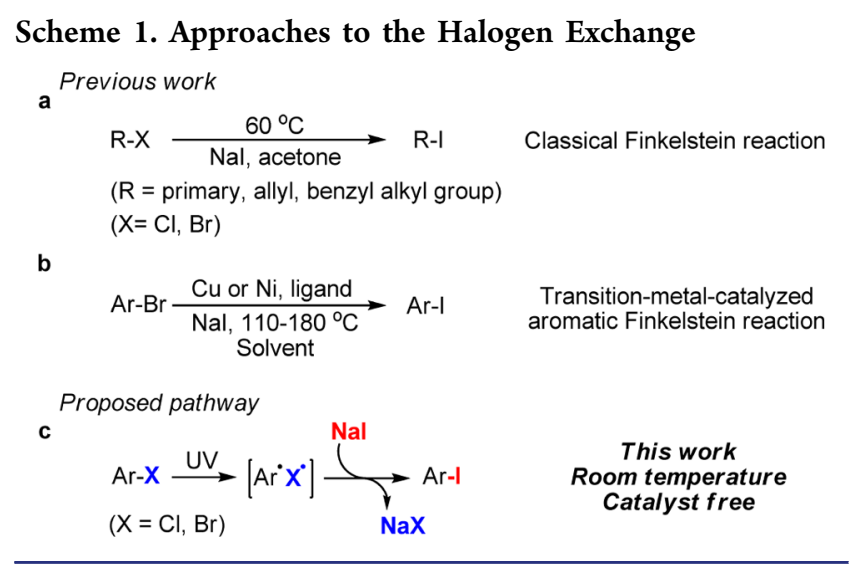

Our investigation started with bromobenzene (1a) and iodized salts at $20{ }^{\circ} \mathrm{C}$ under UV irradiation (UVC, $254 \mathrm{~nm}$ ) at an intensity of $4.0 \mathrm{~mW} \mathrm{~cm}{ }^{-2}$ by using a standard LZC-4V photo-reactor from Luzchem Company (Supporting Information (SI), Figure S1). To cool the reaction vessels, a powerful fan was employed. The airtight quartz tube containing reactants and solvent was evacuated by four freeze-pump-thaw cycles and back-filled with ultra-purified argon (>99.999\%) prior to use. Preliminarily, we analyzed different iodized salts, substrate concentrations, and solvents. As shown in Table 1, the desired iodobenzene (2a) could be obtained in an optimal yield of $69 \%$ by employing 2 equiv of $\mathrm{NaI}$ in $0.5 \mathrm{~mL}$ of acetonitrile after $18 \mathrm{~h}$ photo-reaction. It was noted that the solvent selection is critical

Received: March 27, 2015

Published: June 18, 2015 
Table 1. Selected Results for the Optimization of Reaction Conditions $^{a}$

\begin{tabular}{|c|c|c|c|c|}
\hline entry & iodide salt (mmol) & additive $(\mathrm{mol} \%)^{b}$ & $\mathrm{CH}_{3} \mathrm{CN}(\mathrm{mL})$ & yield $(\%)^{c}$ \\
\hline 1 & $\mathrm{NaI}(0.2)$ & none & 4 & 22 \\
\hline 2 & $\mathrm{NaI}(0.2)$ & none & 2 & 46 \\
\hline 3 & $\mathrm{NaI}(0.2)$ & none & 1 & 56 \\
\hline 4 & $\mathrm{NaI}(0.2)$ & none & 0.5 & 69 \\
\hline 5 & $\mathrm{NaI}(0.2)$ & none & 0.25 & 67 \\
\hline 6 & $\mathrm{NaI}(0.3)$ & none & 0.5 & 66 \\
\hline 7 & $\mathrm{NaI}(0.12)$ & none & 0.5 & 67 \\
\hline 8 & $\mathrm{KI}(0.2)$ & none & 0.5 & 65 \\
\hline 9 & TBAI (0.2) & none & 0.5 & 54 \\
\hline 10 & $\mathrm{NaI}(0.2)$ & $\mathrm{O}_{2}(0.5 \mathrm{~mol} \%)$ & 0.5 & 83 \\
\hline 11 & $\mathrm{NaI}(0.2)$ & $\mathrm{I}_{2}(10 \mathrm{~mol} \%)$ & 0.5 & 91 \\
\hline 12 & none & $\mathrm{I}_{2}(10 \mathrm{~mol} \%)$ & 0.5 & $<5$ \\
\hline $13^{d}$ & $\mathrm{NaI}(0.2)$ & none & 0.5 & NP \\
\hline
\end{tabular}

${ }^{a}$ Reaction conditions: performed with $1 \mathrm{a}(0.1 \mathrm{mmol})$ in argon at room temperature under UV irradiation at an intensity of $4 \mathrm{~mW} \mathrm{~cm}^{-2}$ for 18 h. ${ }^{b}$ In terms of reactant. ${ }^{c}$ Determined by gas chromatography-mass spectrometry (GC-MS) analysis of the crude reaction mixture using chlorobenzene as an internal standard. ${ }^{d}$ Dark reaction. NP $=$ no product.

(SI, Table S1). For instance, acetonitrile is highly transparent in the UV region with a low cut-off wavelength of $190 \mathrm{~nm}$, and $\mathrm{NaI}$ is very soluble in it but the formed $\mathrm{NaBr}$ is not. Therefore, the iodination reaction in acetonitrile can be largely driven toward products. In comparison, methanol, which possesses a similar low UV cut-off wavelength $(205 \mathrm{~nm})$ as acetonitrile, gave inferior yield of $30 \%$ since the produced $\mathrm{NaBr}$ cannot be precipitated from it. On the other hand, due to the high UV cut-off wavelength $(330 \mathrm{~nm})$, acetone cannot be used at 254 $\mathrm{nm}$, and most of the reactants were recovered after the photoreaction in acetone. As shown in SI, Table S2, the iodination yield in acetonitrile solvent decreased gradually with increased cut-off wavelength by using a series of UV long-pass filters. Control experiments further established the requirement of UV light, as no reaction proceeded at all in the dark even under heating. All these features demonstrate that our halide exchange is a photo-induced reaction.

To gain a better understanding of this photo-induced iodination process, we have assessed the side reaction and the effects on the selectivity. During the reaction in acetonitrile, it was found that the iodination reaction competed with the formation of unsubstituted benzene and iodoacetonitrile. SI, Table S3 further indicated that the iodination selectivity decreased gradually with the increase of solvent amount. In the control experiment, no reduced benzene was detected if using hexafluorobenzene as solvent. As shown in SI, Figure S2, bromobenzene has a minor absorption peak around $263 \mathrm{~nm}$ in the UV-visible spectrum, and irradiation at this wavelength leads to homolytic scission of the $\sigma_{\mathrm{C}-\mathrm{Br}}$ bond through the coupling of the $S_{1}\left(\pi, \pi^{*}\right)$ state with the repulsive $n, \sigma^{*}$ triplet state. ${ }^{32}$ The UV light employed in this reaction can introduce sufficient energy $(4.9 \mathrm{eV})$ to excite and break the $\mathrm{C}-\mathrm{Br}$ bonds homolytically (the $\mathrm{C}-\mathrm{Br}$ bond dissociation energy in bromobenzene is $3.4 \mathrm{eV}$ ). Therefore, the major side reaction is a result of hydrogen abstraction from acetonitrile (Sol-H) by the photo-generated phenyl radical. As proposed in Figure 1, the $\mathrm{C}-\mathrm{Br}$ bonds of aryl bromides can be cleaved homolytically

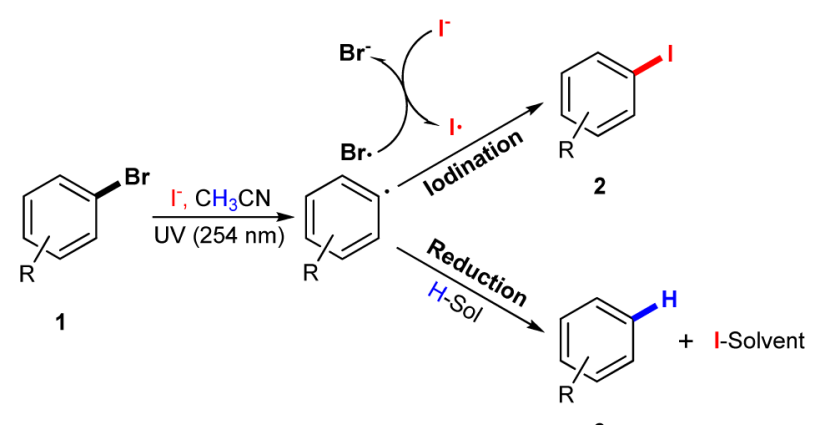

3

Figure 1. Proposed mechanism.

under UV irradiation to generate identical amounts of phenyl radicals and bromine radicals (or atoms). ${ }^{33}$ The produced bromine radicals will further oxidize iodine anions to form iodine radicals, ${ }^{34}$ whereas the phenyl radicals undergo two different reactions. One reaction involves the radical coupling with iodine radicals to yield target product iodobenzene; the other reaction is the hydrogen transfer from solvent as mentioned earlier. To further confirm the hydrogen source, deuterated acetonitrile- $d_{3}$ was used as solvent to carry out the reaction. As expected, the deuterated byproduct benzene- $d_{1}$ was formed. In addition, for some substrates, such as bromobenzene ${ }^{35}$ and aryl bromides with electron-withdrawing groups, an alternative single-electron-transfer mechanism (from $\mathrm{I}^{-}$to $\mathrm{ArBr}$ ) for the formation of the aryl radicals may also be operative in competition with the homolysis route (SI, Figure S3). In the case of 4-chlorophenol, a heterolytic $\mathrm{C}-\mathrm{Cl}$ cleavage (photo- $\mathrm{S}_{\mathrm{N}} 1$ mechanism), in which a phenyl cation is produced and captured by the iodide anion, should be more facile than the homolysis route. ${ }^{36}$

As deduced from the proposed mechanism, the formation of iodine radicals plays an important role in the iodination process. We then set out to further optimize the reaction conditions by altering this process. First, a series of different amount of oxygen, which can enhance the intersystem crossing from the excited singlet state $\left(\mathrm{ArBr}^{*}\right.$, a $\pi, \pi^{*}$ state) to a repulsive triplet state (an $\mathrm{n}, \sigma^{*}$ state) to facilitate the $\mathrm{C}-\mathrm{Br}$ bond homolysis, ${ }^{37}$ was intentionally introduced into the system prior to the reaction. It was found that the injection of small amount of oxygen would give a better yield compared to the pure argon atmosphere conditions (SI, Table S4). The optimal yield of $83 \%$ could be achieved in the presence of $0.5 \mathrm{~mol} \%$ of oxygen (in term of substrate). However, the introduction of gaseous oxygen into the system precisely is hard to control and may also cause undesired oxidized products. To be more practical and selective, an easily handled strategy is needed. Based on the proposed mechanism, the presence of excess iodine radicals during the reaction is anticipated to suppress the phenyl radical reduction side reaction. Consequently, a series of varying amount of iodine was added into the system prior to the reaction under pure argon atmosphere condition, since iodine element can be easily broken into two iodine radicals under UV light. As shown in SI, Table S5, the iodination yield was highly increased in the solution of acetonitrile containing catalytic amount of iodine. Finally, employing $10 \mathrm{~mol} \%$ of iodine element resulted in an optimal yield of 2 a of $91 \%$. The apparent quantum efficiency (iodobenzene produced per 
photon consumed) around $254 \mathrm{~nm}$ was calculated to be ca. $21 \%$ (the calculation details can be found in the SI). To further identify the effects of elemental iodine on the promotion of the reaction, kinetic studies were performed under pure argon, oxygen $(0.5 \mathrm{~mol} \%)$ or iodine $(10 \mathrm{~mol} \%)$ conditions, respectively (Figure 2). Compared to the reaction carried out
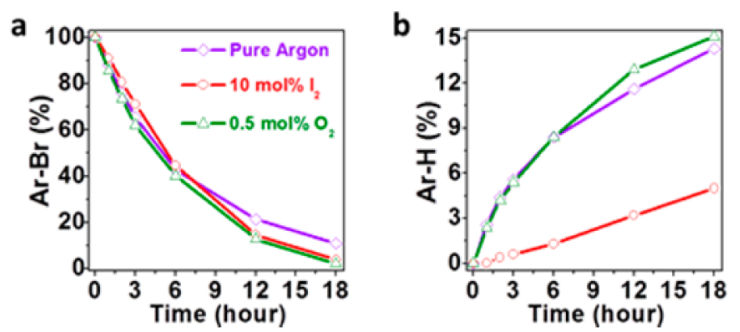

Figure 2. Kinetic studies on the effects of iodine. (a) Time courses of bromobenzene (1a) consumption under different reaction conditions. (b) Plots of byproduct benzene (3a) formation as a function of time. Reaction conditions were as follows: performed with $0.1 \mathrm{mmol}$ of $\mathbf{1 a}$, $0.2 \mathrm{mmol}$ of $\mathrm{NaI}$, and $0.5 \mathrm{~mL}$ of $\mathrm{CH}_{3} \mathrm{CN}$ at room temperature upon UV irradiation in pure argon (blue curve), argon with $10 \mathrm{~mol} \%$ of $\mathrm{I}_{2}$ (red curve), or argon with $0.5 \mathrm{~mol} \%$ of $\mathrm{O}_{2}$ (green curve), respectively.

under pure argon conditions, the introduction of $10 \mathrm{~mol} \%$ of iodine element can significantly suppress the formation of unsubstituted byproduct (Figure $2 b$, red curve).

With the optimized conditions in hand, we subjected a series of aryl and heteroaryl bromides 1 to the photo-induced aromatic halogen exchange reaction (Scheme 2). In general, the clean procedure typically delivered the corresponding aryl iodide $\mathbf{2}$ in yields ranging from $56 \%$ to $93 \%$. Due to the mild conditions without any metal catalysts or bases, the products were isolated cleanly with minimal decomposition. The aryl bromide with diverse substituents including either electronwithdrawing groups or electron-donating groups gave very good yields $(\mathbf{2} \mathbf{b}-\mathbf{2 i})$. The polar functional groups are highly tolerated including ester, nitrile, and alcohol $(\mathbf{2 j}-\mathbf{2 l})$. Polycyclic and heterocyclic bromides and $\mathrm{N}-\mathrm{H}$-containing substrates such as 1-bromonaphthalene, 5-bromopyrimidine, and 5bromoindole were also iodinated cleanly and in good yield; in other cases the presence of a second benzene rings caused no problems. For the dibromo-substituted benzene ring, both para- and meta-dibromobenzene could be converted into the corresponding diiodobenzene with good yield, respectively (2s, $2 t)$. Several of the substrates $(2 \mathbf{p}, \mathbf{2 u}-\mathbf{2 x})$ required a more dilute solution $(1.5 \mathrm{~mL})$ for optimal yields. In addition, we conducted a large-scale reaction, and the results revealed that our system was effective on the gram scale $(\mathbf{2 b})$.

Further experiments demonstrated that the photo-driven strategy could be extended to halogen exchange in aryl chlorides (2a, $\mathbf{2} \mathbf{b}$, and $\mathbf{2 g}$ in Scheme 2 ) and vinyl bromides (eq 1) in a satisfactory yield under identical conditions.

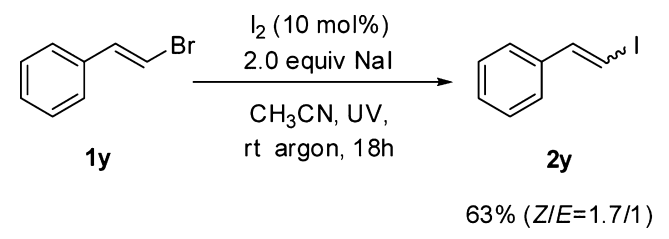

Interestingly, the iodination of (E)-1-bromo-2-phenylethene gave a ( $Z$ )-1-iodo-2-phenylethene predominantly. It is also noted that the addition of $\mathrm{I}_{2}$ to the carbon-carbon double
Scheme 2. Iodination of Various Aryl Bromides and Chlorides $^{a, b}$

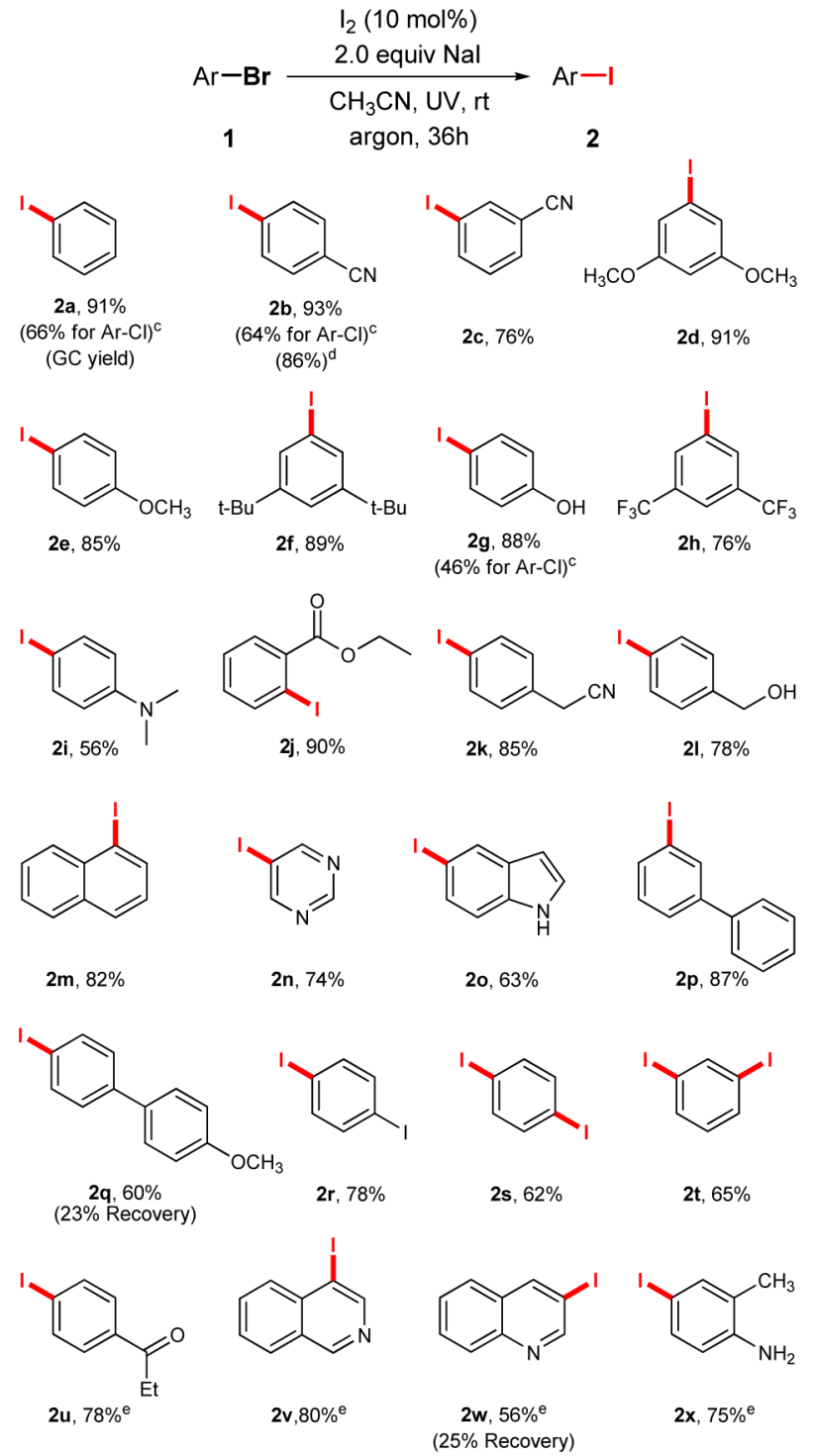

${ }^{a}$ Reaction conditions: aryl bromide $1(0.1 \mathrm{mmol}), \mathrm{NaI}(0.2 \mathrm{mmol}), \mathrm{I}_{2}$ $(0.01 \mathrm{mmol})$, and $\mathrm{CH}_{3} \mathrm{CN}(0.5 \mathrm{~mL})$ at $20^{\circ} \mathrm{C}$ in argon under UV light for $36 \mathrm{~h}$. ${ }^{b}$ Yield of isolated product is given. ${ }^{\text {Codination of aryl }}$ chlorides to the corresponding iodides. ${ }^{d}$ Gram scale reaction (6 $\mathrm{mmol}$ ). ${ }^{e}$ Performed in $1.5 \mathrm{~mL}$ of $\mathrm{CH}_{3} \mathrm{CN}$.

bond was not observed during the reaction under photoirradiation at $20{ }^{\circ} \mathrm{C}$, which will occur above $40{ }^{\circ} \mathrm{C}$ in our system even under darkness. Consequently, the temperature must be kept below $20{ }^{\circ} \mathrm{C}$ during the workup to avoid the formation of $\alpha, \beta$-addition products.

In summary, we have first developed a photo-promoted metal-catalyst-free protocol for the conversion of aryl, heteroaryl, and vinyl bromides (or chlorides) into the corresponding iodides. Significantly, this strategy allows the direct activation of carbon-halogen bonds and the electron transfer between halide species. This work provides a practical and greener protocol to not only synthesize aryl iodide compounds but also generate reactive aryl radicals under mild and metal-catalyst-free conditions, which are very useful in organic synthesis. 


\section{ASSOCIATED CONTENT}

\section{S Supporting Information}

Experimental procedures and analytical data. The Supporting Information is available free of charge on the ACS Publications website at DOI: $10.1021 /$ jacs.5b03220.

\section{AUTHOR INFORMATION}

\section{Corresponding Authors}

*zetian.mi@mcgill.ca

*cj.li@mcgill.ca

\section{Author Contributions}

${ }^{\S}$ L.L. and W.L. contributed equally.

\section{Notes}

The authors declare no competing financial interest.

\section{ACKNOWLEDGMENTS}

This work was financially supported by the Canada Research Chair (Tier 1) foundation, the Natural Sciences and Engineering Research Council of Canada, the Fonds de recherchésur la nature et les technologies, Canada Foundation for Innovation (CFI), and McGill University.

\section{REFERENCES}

(1) Tilly, D.; Chevallier, F.; Mongin, F.; Gros, P. C. Chem. Rev. 2014, 114, 1207.

(2) Knochel, P.; Dohle, W.; Gommermann, N.; Kneisel, F. F.; Kopp, F.; Korn, T.; Sapountzis, I.; Vu, V. A. Angew. Chem., Int. Ed. 2003, 42, 4302.

(3) (a) Bunnett, J. F.; Zahler, R. Chem. Rev. 1951, 49, 273. (b) Hartwig, J. F. Synlett 2006, 9, 1283.

(4) Bolm, C.; Hildebrand, J. P.; Muñiz, K.; Hermanns, N. Angew. Chem., Int. Ed. 2001, 40, 3284.

(5) (a) Evano, G.; Blanchard, N.; Toumi, M. Chem. Rev. 2008, 108, 3054. (b) Ma, D.; Cai, Q. Acc. Chem. Res. 2008, 41, 1450.

(6) (a) Bunevičius, R.; Kažanavičius, G.; Žalinkevičius, R.; Prange, A. J. N. Engl. J. Med. 1999, 340, 424. (b) Roberts, C. G.P.; Ladenson, P. W. Lancet 2004, 363, 793.

(7) Pimlott, S. L.; Sutherland, A. Chem. Soc. Rev. 2011, 40, 149.

(8) Hallouard, F.; Anton, N.; Choquet, P.; Constantinesco, A.; Vandamme, T. Biomaterials 2010, 31, 6249.

(9) Seevers, R. H.; Counsell, R. E. Chem. Rev. 1982, 82, 575.

(10) Barluenga, J.; González, J. M.; Martín, M. A. G.; Campos, P. J.; Asensio, G. J. Org. Chem. 1993, 58, 2058.

(11) Takagi, K.; Hayama, N.; Inokawa, S. Bull. Chem. Soc. Jpn. 1980, $53,3691$.

(12) Yang, S. H.; Li, C. S.; Cheng, C. H. J. Org. Chem. 1987, 52, 691.

(13) Sheppard, T. D. Org. Biomol. Chem. 2009, 7, 1043.

(14) Kalyani, D.; Dick, A. R.; Anani, W. Q.; Sanford, M. S. Org. Lett. 2006, 8, 2523

(15) Mei, T. S.; Giri, R.; Maugel, N.; Yu, J. Q. Angew. Chem., Int. Ed. 2008, 47, 5215.

(16) Dudnik, A. S.; Chernyak, N.; Huang, C.; Gevorgyan, V. A. Angew. Chem., Int. Ed. 2010, 49, 8729.

(17) Newman, S. G.; Howell, J. K.; Nicolaus, N.; Lautens, M. J. Am. Chem. Soc. 2011, 133, 14916.

(18) Wang, X. C.; Hu, Y.; Bonacorsi, S.; Hong, Y.; Burrell, R.; Yu, J. Q. J. Am. Chem. Soc. 2013, 135, 10326.

(19) Klapars, A.; Buchwald, S. L. J. Am. Chem. Soc. 2002, 124, 14844.

(20) Casitas, A.; Canta, M.; Solà, M.; Costas, M.; Ribas, X. J. Am. Chem. Soc. 2011, 133, 19386.

(21) Cant, A. A.; Bhalla, R.; Pimlott, S. L.; Sutherland, A. Chem. Commun. 2012, 48, 3993.

(22) Chen, M.; Ichikawa, S.; Buchwald, S. L. Angew. Chem., Int. Ed. 2015, 54, 263.

(23) Sun, C. L.; Shi, Z. J. Chem. Rev. 2014, 114, 9219.
(24) Prier, C. K.; Rankic, D. A.; MacMillan, D. W. C. Chem. Rev. 2013, 113, 5322

(25) Li, L.; Li, G. D.; Yan, C.; Mu, X. Y.; Pan, X. L.; Zou, X. X.; Wang, K. X.; Chen, J. S. Angew. Chem., Int. Ed. 2011, 50, 8299.

(26) Li, L.; Cai, Y. Y.; Li, G. D.; Mu, X. Y.; Wang, K. X.; Chen, J. S. Angew. Chem., Int. Ed. 2012, 51, 4702.

(27) Uyeda, C.; Tan, Y.; Fu, G. C.; Peters, J. C. J. Am. Chem. Soc. 2013, 135, 9548.

(28) Becerril, M. R.; Mahé, O.; Drouin, M.; Majewski, M. B.; West, J. G.; Wolf, M. O.; Sammis, G. M.; Paquin, J. F. J. Am. Chem. Soc. 2014, 136, 2637.

(29) Li, L.; Fan, S.; Mu, X.; Mi, Z.; Li, C. J. J. Am. Chem. Soc. 2014, 136, 7793.

(30) Tellis, J. C.; Primer, D. N.; Molander, G. A. Science 2014, 345, 433.

(31) Liu, W.; Li, L.; Li, C. J. Nat. Commun. 2015, 6, No. 6526.

(32) (a) Zhang, H.; Zhu, R. S.; Wang, G. J.; Han, K. L.; He, G. Z.; Lou, N. Q. J. Chem. Phys. 1999, 110, 2922. (b) Tang, B.; Zhu, R.; Tang, Y.; Ji, L.; Zhang, B. Chem. Phys. Lett. 2003, 381, 617.

(33) Rasmusson, M.; Lindh, R.; Lascoux, N.; Tarnovsky, A. N.; Kadi, M.; Kühn, O.; Sundström, V.; Åkesson, E. Chem. Phys. Lett. 2003, 367, 759.

(34) (a) Popov, A. I.; Geske, D. H. J. Am. Chem. Soc. 1958, 80, 1340. (b) Lin, C. Y.; Coote, M. L.; Gennaro, A.; Matyjaszewski, K. J. Am. Chem. Soc. 2008, 130, 12762.

(35) (a) Rehm, D.; Weller, A. Isr. J. Chem. 1970, 8, 259. (b) M'Halla, F.; Pinson, J.; Saveant, J. M. J. Am. Chem. Soc. 1980, 102, 4120. (c) Weller, A. Z. Phys. Chem. Neue Folge 1982, 133, 93.

(36) (a) Fagnoni, M.; Albini, A. Acc. Chem. Res. 2005, 38, 713. (b) Dichiarante, V.; Fagnoni, M. Synlett 2008, 6, 787. (c) Lazzaroni, S.; Protti, S.; Fagnoni, M.; Albini, A. Org. Lett. 2009, 11, 349.

(37) (a) Kearns, D. R. Chem. Rev. 1971, 71, 395. (b) McLean, A. J.; McGarvey, D. J.; Truscott, T. G.; Lambert, C. R.; Land, E. J. J. Chem. Soc., Faraday Trans. 1990, 86, 3075. 